OPTICA ACTA, 1985 , VOL. 32 , NO. $3,345-355$

\title{
Binomial states of the quantized radiation field
}

\author{
D. STOLER
}

AT\&T Bell Laboratories, Summit, New Jersey 07901, U.S.A.

\section{B. E. A. SALEH}

Department of Electrical and Computer Engineering, Univerisity of Wisconsin, Madison, Wisconsin 53706, U.S.A. and Columbia Radiation Laboratory, Department of Electrical Engineering, New York 10027, U.S.A.

\section{C. TEICH}

Columbia Radiation Laboratory, Department of Electrical Engineering, New York 10027, U.S.A.

(Received 1 October 1984)

\begin{abstract}
We introduce the binomial state of the quantized radiation field, which is a state that yields a binomial counting probability distribution. It reduces to the coherent state and to the number state in different limits. The binomal state is quantum mechanical in nature, and we show that it produces light that is antibunched, sub-poissonian, and squeezed for certain parameter ranges. A mixed binomial state is also defined. Particular attention is devoted to the mixed Bernoulli state which is an important special case. The properties of this mixed state indicate that squeezing might be present in individual atomic emissions.
\end{abstract}

\section{Introduction}

The number state $|n\rangle$ and the coherent state $|\alpha\rangle$ are well-studied states of the radiation field that have been dealt with extensively since the early days of quantum optics [1]. There are many ways to generate these states. As an example, a single atom in its first excited state, in the absence of external interactions, is generally assumed to radiate an $|n=1\rangle$ state. A classical charge distribution, on the other hand, radiates a coherent state [1].

We introduce the binomial state which interpolates between the number state and the coherent state. It partakes of the properties of both and reduces to each in different limits. The binomial state $|\eta, M\rangle$ is a linear combination of the $n$ states $|0\rangle,|1\rangle, \ldots,|M\rangle$ with coefficients chosen such that the photon-counting probability distribution is binomial with mean $\eta M$. This is similar to the coherent state $|\alpha\rangle$ which is a linear combination of all $|n\rangle$ states with coefficients chosen such that the photoncounting distribution is poissonian. Binomial photon-counting distributions have been discussed in the literature [2]. Like the generalized coherent states for which coefficients of its $n$ state expansion are allowed to have additional arbitrary phases, a generalized binomial state may be similarly defined. The binomial state may be generated when each of $M$ photons is emitted with probability $\eta$, i.e., when an excited molecule undergoes $M$ level vibrational relaxation under certain conditions. 
Important special cases are the generalized and mixed Bernoulli states $(M=1)$.

In $\S 2$ we discuss the properties of the binomial state. The behaviour of the mixed Bernoulli state, and some considerations about how it might be produced, are dealt with in $\S 3$. The discussion is contained in $\S 4$.

\section{The binomial state}

\subsection{Mathematical properties}

We define a pure state of a single mode of the electromagnetic field for which the photon-number probability density is binomial, i.e.

$$
|\eta, M\rangle=\sum_{n=0}^{M} \beta_{n}^{M}|n\rangle
$$

where

$$
\beta_{n}^{M}=\left[\left(\begin{array}{l}
M \\
n
\end{array}\right) \eta^{n}(1-\eta)^{M-n}\right]^{1 / 2} .
$$

In equation (1), $|n\rangle$ is the number state of the field mode and $\{\eta,(1-\eta)\}$ are the probabilities of the two possible outcomes of a Bernoulli trial. In the present context we see that $\left|\beta_{n}^{M}\right|^{2}$ is the probability for the production of $n$ photons when there are $M \geqslant n$ independent ways to produce a photon. The binomial states defined by equation (1) are normalized since

$$
\langle\eta, M \mid \eta, M\rangle=\sum_{n=0}^{M}\left(\begin{array}{c}
M \\
n
\end{array}\right) \eta^{n}(1-\eta)^{M-n}=1 .
$$

The mean photon number in a binomial state is given by

$$
\begin{aligned}
\langle\eta, M|\hat{n}| \eta, M\rangle & =\sum_{n=0}^{M} n\left|\beta_{n}^{M}\right|^{2} \\
& =\sum_{n=0}^{M} n \frac{M !}{n !(M-n) !}\left(\frac{\eta}{1-\eta}\right)^{n}(1-\eta)^{M} \\
& =\eta M,
\end{aligned}
$$

where $\hat{n}$ is the number operator obeying the relation $\hat{n}|n\rangle=n|n\rangle$. This is as expected for the binomial distribution. Defining $\sigma=\eta /(1-\eta)$, we obtain

$$
\begin{gathered}
\langle\hat{n}\rangle_{\eta, M}=\frac{M \sigma}{1+\sigma}=\eta M, \\
\left\langle\hat{n}^{2}\right\rangle_{\eta, M}=\frac{M \sigma(M \sigma+1)}{(1+\sigma)^{2}}=(\eta M)^{2}+\eta(1-\eta) M, \\
(\Delta n)^{2} \equiv\langle\Delta \hat{n}\rangle_{\eta, M}^{2}=\left\langle\hat{n}^{2}\right\rangle_{\eta, M}-\langle\hat{n}\rangle_{\eta, M}^{2}=M \sigma /(1+\sigma)^{2}=\eta(1-\eta) M .
\end{gathered}
$$

The ratio of variance to mean (Fano factor) is given by

$$
(\Delta n)^{2} /\langle\hat{n}\rangle_{\eta, M}=1-\eta,
$$

indicating the sub-poissonian nature of the binomial distribution. The bunching parameter $\Delta=(\Delta n)^{2}-\langle\hat{n}\rangle$ for the binomial state is

$$
\Delta_{\eta, M}=-\eta^{2} M<0 .
$$


The normalized second-order correlation function $g^{(2)}(0)$ for $|\eta, M\rangle$ may be expressed as

$$
g_{\eta, M}^{(2)}(0)=1-1 / M=1-\eta /\langle\hat{n}\rangle_{\eta, M}
$$

The quantity $g^{(2)}(0)$ is defined as $\left\langle\hat{a}^{\dagger} \hat{a}^{\dagger} \hat{a} \hat{a}\right\rangle \mid\left\langle\hat{a}^{\dagger} \hat{a}\right\rangle^{2}$ where $\hat{a}$ and $\hat{a}^{\dagger}$ are the lowering and raising operators for the field mode of interest, respectively. Since $g^{(2)}(0)<1$, these states are antibunched, but less so than the number states. Antibunching and sub-poissonian behaviour always accompany each other for single-mode timeindependent fields [3].

For $\eta=0$ and 1 , with $M$ finite, the binomial states reduce to the vacuum state $|0\rangle$ and the number state $|n=M\rangle$ respectively. In the limit $\eta \rightarrow 0, M \rightarrow \infty$ (with $\eta M=$ constant $=\alpha), \Delta \rightarrow 0$. The binomial state then approaches a coherent state $|\alpha\rangle$. Since the $\beta_{n}^{M}$ have been chosen to be real, the most general coherent state is not obtained, but rather one with a real value of the amplitude parameter $\alpha$. Had the coefficients in equation (1) been chosen to be complex, i.e. $c_{n}^{M}=\beta_{n}^{M} \operatorname{exp~i} \theta_{n}$ where $\left\{\theta_{n}\right\}$ is a set of arbitrary real numbers, the limit would yield the generalized coherent state of Titulaer and Glauber [4]. In $\S 3$ we will consider the generalized Bernoulli state $|\eta, \phi\rangle$ which is a special case of the binomial state $(M=1)$ with complex $\beta$.

It is interesting to point out that the binomial states cannot have the minimum uncertainty product because they contain a finite number of $|n\rangle$ states for any given $M$ and the minimality condition cannot be satisfied by any such finite combination [5]. The uncertainty product for the $|\eta, M\rangle$ states will of course approach the minimum value in the limit $\eta \rightarrow 0, M \rightarrow \infty$ since the limiting case, a coherent state, is a minimal packet. Similarly, when $\eta=0$ the binomial state reduces to the vacuum state, which is also a coherent state and a minimal packet. We have derived the expansion for the coherent state in the binomial-state basis which may be of some interest (see Appendix).

The binomial states display antibunching and sub-poissonian behaviour and yet cannot have a minimum uncertainty product. Because of this distinctly nonclassical behaviour, it is of interest to examine the squeezing properties of these states. To investigate this we first examine the effects of the raising and lowering operators on $|\eta, M\rangle$. Noting that $\hat{a}$ and $\hat{a}^{\dagger}$ obey the boson commutation relation $\left[\hat{a}, \hat{a}^{\dagger}\right]=1$, we have

$$
\hat{a}|\eta, M\rangle=\sum_{n=0}^{M} \beta_{n}^{M} n^{1 / 2}|n-1\rangle .
$$

Noting the identity

$$
n^{1 / 2} \beta_{n}^{M}=(\eta M)^{1 / 2} \beta_{n-1}^{M-1}
$$

equation (10) yields

$$
\hat{a}|\eta, M\rangle=(n M)^{1 / 2} \sum_{n=0}^{M} \beta_{n-1}^{M-1}|n-1\rangle .
$$

The summation in equation (12) actually begins with $n=1$, since the $n=0$ term vanishes. Changing the summation index in equation (12) to $l=n-1$ finally leads to

$$
\begin{aligned}
\hat{a}|\eta, M\rangle & =(\eta M)^{1 / 2} \sum_{l=0}^{M-1} \beta_{l}^{M-1}|l\rangle \\
& =(\eta M)^{1 / 2}|\eta, M-1\rangle .
\end{aligned}
$$


Therefore $\hat{a}$ acts as a kind of lowering operator for the $|\eta, M\rangle$ state, since $\eta M=\langle\hat{n}\rangle_{\eta, M}$. The analagous result for $\hat{a}^{\dagger}$ is however more complicated. It turns out that

$$
\hat{a}^{\dagger}|\eta, M\rangle=[\eta(M+1)]^{-1 / 2} \hat{n}|\eta, M+1\rangle
$$

so that $\hat{a}^{\dagger}$ is not a raising operator for $|\eta, M\rangle$. Using equations (13) and (14), each $n$ times, permits us to derive the following expressions easily:

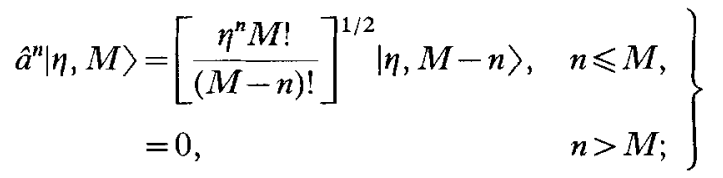

$$
\begin{aligned}
& \hat{a}^{\dagger n}|\eta, M\rangle=\left[\frac{M !}{\eta^{n}(M+n) !}\right]^{1 / 2} \hat{n}(\hat{n}-1) \ldots(\hat{n}-n+1)|\eta, M+n\rangle .
\end{aligned}
$$

The average values are then obtained quite directly by using equation (15):

$$
\left\langle\eta, M\left|\hat{a}^{n}\right| \eta, M\right\rangle=\left[\frac{\eta^{n} M !}{(M-n) !}\right]^{1 / 2}\langle\eta, M \mid \eta, M-n\rangle .
$$

Taking the complex conjugate of equation (17) yields

$$
\left\langle\eta, M\left|\hat{a}^{\dagger n}\right| \eta, M\right\rangle=\left[\frac{\eta^{n} M !}{(M-n) !}\right]^{1 / 2}\langle\eta, M-n \mid \eta, M\rangle .
$$

Since $\beta_{n}^{W}$ is real, we have $\langle\eta, . M-n \mid \eta, . I\rangle=\langle\eta, M \mid \eta, M-n\rangle$. This results in the equality of $\left\langle\hat{a}^{n}\right\rangle_{\eta, M}$ and $\left\langle\hat{a}^{\dagger n}\right\rangle_{\eta, M}$. Equations (17) and (18) (for $n=2$ ), along with equation (3), are used to evaluate the variances of the field quadratures to determine the squeezing properties of the $|\eta, M\rangle$ states.

Defining the quadrature (position/momentum type) operators in the usual fashion gives

$$
\begin{aligned}
& \hat{x}=2^{-1 / 2}\left(\hat{a}+\hat{a}^{\dagger}\right), \\
& \hat{p}=-i 2^{-1 / 2}\left(\hat{a}-\hat{a}^{\dagger}\right) .
\end{aligned}
$$

Equations (17) and (18) provide

$$
\begin{aligned}
\langle\eta, M|\hat{x}| \eta, M\rangle & =2^{1 / 2}\langle\eta, M|\hat{a}| \eta, M\rangle \\
& =(2 \eta M)^{1 / 2}\langle\eta, M \mid \eta, M-1\rangle, \\
\langle\eta, M|\hat{p}| \eta, M\rangle & =0 .
\end{aligned}
$$

The second moments are

$$
\begin{aligned}
& \left\langle\eta, M\left|\hat{x}^{2}\right| \eta, M\right\rangle=\left\langle\eta, M\left|\hat{a}^{2}\right| \eta, M\right\rangle+\eta M+\frac{1}{2}, \\
& \left\langle\eta, M\left|\hat{p}^{2}\right| \eta, M\right\rangle=-\left\langle\eta, M\left|\hat{a}^{2}\right| \eta, M\right\rangle+\eta M+\frac{1}{2} .
\end{aligned}
$$

Equation (17) for $n=2$ provides

$$
\left\langle\eta, M\left|\hat{a}^{2}\right| \eta, M\right\rangle=\left[\eta^{2} M(M-1)\right]^{1 / 2}\langle\eta, M \mid \eta, M-2\rangle .
$$

Finally, we find the following expressions for the variances:

$$
(\Delta x)^{2}=\left[\eta^{2} M(M-1)\right]^{1 / 2}\langle\eta, M \mid \eta, M-2\rangle-2 \eta M\langle\eta, M \mid \eta, M-1\rangle^{2}+\eta M+\frac{1}{2},
$$




$$
(\Delta p)^{2}=\left\langle\eta, M\left|\hat{p}^{2}\right| \eta, M\right\rangle=-\left[\eta^{2} M(M-1)\right]^{1 / 2}\langle\eta, M \mid \eta, M-2\rangle+\eta M+\frac{1}{2} .
$$

The squeezing properties of the binominal states are therefore determined by the matrix elements $\langle\eta, M \mid \eta, M-1\rangle$ and $\langle\eta, M \mid \eta, M-2\rangle$, as functions of $\eta$ and $M$. These matrix elements are easily expressed as finite series which can be evaluated numerically. The general result is

$$
\left\langle\eta, M \mid \eta, . M^{\prime}\right\rangle=\sum_{n=0}^{M} \beta_{n}^{M} \beta_{n}^{W^{\prime}},
$$

where $M_{<}$is the smaller of $M$ and $M^{\prime}$.

In the next subsection we present the results of numerical calculations demonstrating the squeezing patterns of the $|\eta, M\rangle$ states.

\subsection{Parametric study of squeezing in the pure binomial state}

The pure binomial state is indeed squeezed under certain conditions. This is illustrated graphically in figure 1 where we plot $(\Delta p)^{2}$ versus $(\Delta x)^{2}$ for the state $|\eta, M\rangle$. The point $\left(\frac{1}{2}, \frac{1}{2}\right)$ represents a nonsqueezed minimum uncertainty state, viz. the coherent state. The vertical and horizontal dashed lines are the squeezing boundaries for $(\Delta x)^{2}$ and $(\Delta p)^{2}$, respectively (e.g. points to the left of the vertical dashed line are squeezed in $x)$. The dashed hyperbola $(\Delta p)^{2}(\Delta x)^{2}=\frac{1}{4}$ represents the locus of minimum uncertainty states (the $|r, \alpha\rangle$ states), all of which exhibit squeezing, with the exception of the coherent state [6]. Points lying on the hyperbola away from

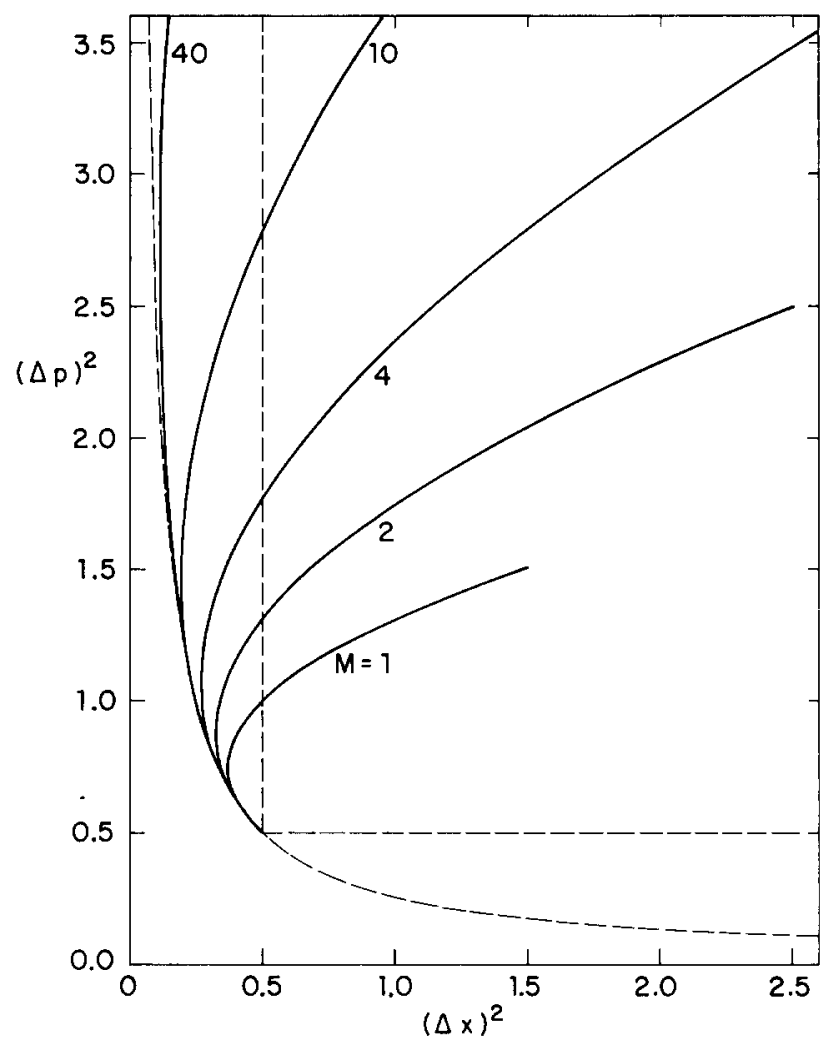

Figure 1. $(\Delta p)^{2}$ versus $(\Delta x)^{2}$ for the binomial state $|\eta, M\rangle$. 
$\left(\frac{1}{2}, \frac{1}{2}\right)$ are both squeezed and minimum uncertainty, whereas those lying between the hyperbola and the dashed lines are squeezed but not minimum uncertainty. The solid curves represent explicit results for the binomial state with $M=1,2,4,10$, and 40. $M=1$ represents the Bernoulli state. 'These curves all intersect $\left(\frac{1}{2}, \frac{1}{2}\right)$ for $\eta=0$ (where the binomial state degenerates to the vacuum state), and spiral away from this point as $\eta$ increases. For $\eta$ sufficiently low, the binomial states exhibit squeezing as well as almost minimum uncertainty behaviour (as we have already pointed out, these states cannot be precisely minimal). As $\eta$ increases they maintain their squeezed properties while losing their low uncertainty properties. Finally, above a certain threshold $\eta_{\mathrm{th}}(M)$, the curves fall to the right of the vertical dashed line indicating nonsqueezed, non-minimal behaviour. The maximum squeezing attainable increases with $M$ and is achieved for an intermediate value of $\eta$. For example when $M=40$, maximal squeezing is achieved for $\eta \simeq 0 \cdot 8$. At this value $(\Delta x)^{2}=0 \cdot 12$ and the minimum uncertainty product $(\Delta p)^{2}(\Delta x)^{2} \simeq 0 \cdot 31$. Of course, as $\eta \rightarrow 1$ for arbitrary $M$, the binomial state $|1, M\rangle$ approaches the number state $|n=M\rangle$, for which $(\Delta p)^{2}=(\Delta x)^{2}=M+\frac{1}{2}$ (the number-state limit is evident in figure 1 for $M=1,2)$. Since for $|n=40\rangle$ we have $(\Delta x)^{2}=40 \cdot 5$ and $(\Delta p)^{2}(\Delta x)^{2}=1640 \cdot 25$, it is clear that the loss of squeezing and low uncertainty product sets on rapidly as $\eta$ increases from 0.8 to 1.0 . We recall that the results presented here are derived for real coefficients $\beta_{n}^{M}$. For complex coefficients, the mean momentum $\langle\eta, M|p| \eta, M\rangle \neq 0$ and $p$ will exhibit squeezing for certain choices of the phases.

When $M=1$ the pure binomial state reduces to the pure Bernoulli state which is squeezed for $\eta<\frac{1}{2}$ and maximally squeezed for $\eta=\frac{1}{4}$. Explicit results for the squeezing have been derived for complex coefficients. The properties of the resulting generalized Bernoulli state will be discussed in detail in $\S 3$.

\subsection{The generalized and mixed binomial state}

The most general pure state whose counting distribution is binomial is obtained by multiplying the coefficients $\beta_{n}^{M}$ by arbitrary phase factors so that

$$
\begin{gathered}
\left.\left|\eta, M,\left\{\theta_{n}^{\prime}\right\rangle=\sum_{n=0}^{M} c_{n}^{M}\right| n\right\rangle, \\
c_{n}^{M}=\beta_{n}^{M} \exp \left(i \theta_{n}\right) .
\end{gathered}
$$

The properties of such a state will be dependent on the choice of phases. If the phases are allowed to be random, the result is a mixed state with a density operator whose diagonal elements in an $n$ state representation $\rho_{n n}$ are given by the binomial distribution. Because of the presence of so many parameters in equation (29), it is difficult to determine regions in this $M$ dimensional parameter space where squeezing occurs.

\section{Squeezing properties of the Bernoulli state}

Consider electromagnetic radiation in a mixed state whose density operator is defined by the number-state representation matrix elements

$$
\left.\begin{array}{rl}
\langle 1|\rho| 1\rangle & =\eta, \\
\langle 0|\rho| 0\rangle & =1-\eta, \\
\langle 1|\rho| 0\rangle & =R[\eta(1-\eta)]^{1 / 2} \exp (\mathrm{i} \phi), \\
\langle 0|\rho| 1\rangle & =R[\eta(1-\eta)]^{1 / 2} \exp (-\mathrm{i} \phi),
\end{array}\right\}
$$


with all other elements being zero. We refer to this state as the mixed Bernoulli state. When $R=1$, it becomes the generalized Bernoulli state,

$$
|\eta, \phi\rangle=(1-\eta)^{1 / 2}|0\rangle+\eta^{1 / 2} \exp (\mathrm{i} \phi)|1\rangle .
$$

When $\phi=0$, the state becomes the Bernoulli state (which is a special case of the binomial state discussed in detail in $§ 2$ ).

It is apparent that mixed Bernoulli-state light has a Bernoulli photon-counting distribution (which is sub-poissonian) and is therefore also antibunched. The squeezing properties of the mixed Bernoulli state depend on the parameters $\eta, \phi$, and $R$. Expressions for the position/momentum mean and variance turn out to be

$$
\begin{aligned}
\langle\hat{x}\rangle & =R[2 \eta(1-\eta)]^{1 / 2} \cos \phi, \\
\langle\hat{p}\rangle & =R[2 \eta(1-\eta)]^{1 / 2} \sin \phi, \\
(\Delta x)^{2} & =\frac{1}{2}+\eta-R^{2}\left[2 \eta(1-\eta) \cos ^{2} \phi\right], \\
(\Delta p)^{2} & =\frac{1}{2}+\eta-R^{2}\left[2 \eta(1-\eta) \sin ^{2} \phi\right] .
\end{aligned}
$$

\subsection{The pure generalized Bernoulli state}

We first examine the conditions for squeezing in the special case $R=1$, i.e. for the pure state. For $\phi=m \pi, m=0,1,2, \ldots$, the results reduce to the ordinary Bernoulli state $(\langle p\rangle=0)$ presented in figure $1(M=1)$. For $\phi=\pi / 2+m \pi$, the roles of $x$ and $p$ interchange, with $p$ exhibiting squeezing and $\langle\hat{x}\rangle=0$. For $\phi=\pi / 4+m \pi / 2$, there is complete symmetry between $x$ and $p$ such that

$$
(\Delta p)^{2}=(\Delta x)^{2}=\frac{1}{2}+\eta^{2},
$$

and neither quadrature component is squeezed.

Equations (33) and (34) [with $R=1$ ] may be combined in any number of ways, leading to several simple but useful expressions:

$$
\begin{gathered}
(\Delta p)^{2}+(\Delta x)^{2}=1+2 \eta^{2}, \\
(\Delta p)^{2}-(\Delta x)^{2}=2 \eta(1-\eta) \cos 2 \phi, \\
(\Delta p)^{2}(\Delta x)^{2}=\frac{1}{4}+2 \eta^{3}+[\eta(1-\eta)]^{2} \sin ^{2} 2 \phi .
\end{gathered}
$$

In figure 2 we plot equations (33) and (34), with $R=1$ (solid curves) along with equation (36) (diagonal solid lines). Parametric values for $\eta$ and $\phi$ are given in the figure. Also shown are the squeezing boundaries (dashed vertical and horizontal lines) and the minimum-uncertainty hyperbola. Squeezing is maximized for $\phi=0$ or $\pi / 2$, and is altogether absent for $\phi=\pi / 4$. As $\phi$ increases from 0 toward $\pi / 4$, both the range of $\eta$ over which squeezing occurs, and the maximum squeezing attainable, decrease.

Simple analytical expressions can be derived for these quantities. The maximum value of $\eta$ below which squeezing is obtained (denoted $\eta_{x, p}$ ) is determined from the definition $(\Delta x)^{2}<\frac{1}{2}$ or $(\Delta p)^{2}<\frac{1}{2}$, which leads to

$$
\begin{aligned}
& \eta_{x}=1-\left(2 \cos ^{2} \phi\right)^{-1} \\
& \eta_{p}=1-\left(2 \sin ^{2} \phi\right)^{-1}
\end{aligned}
$$




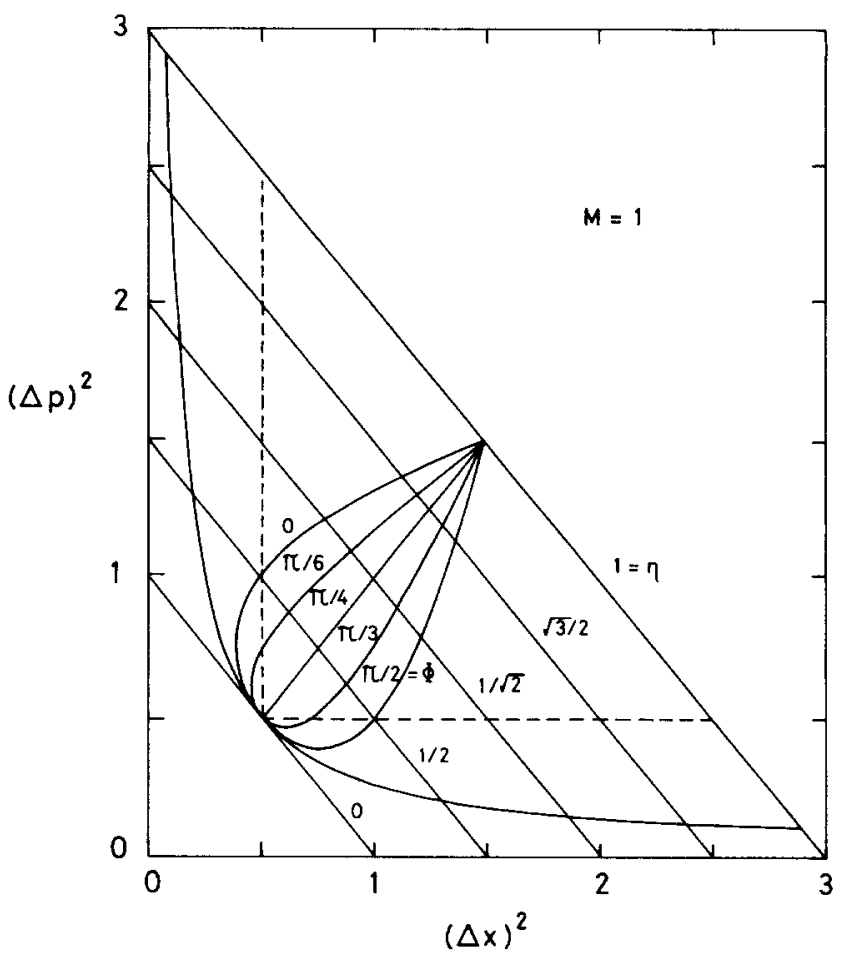

Figure 2. $(\Delta p)^{2}$ versus $(\Delta x)^{2}$ for the generalized Bernoulli state $|\eta, \phi\rangle$.

Of course, $\eta$ is lower bounded by 0 so there may be no range where $p$ or $x$ is squeezed (e.g. for $\phi=0, p$ is never squeezed). The optimal value of $\eta$ (denoted $\eta_{x o, p o}$ ) for maximal squeezing turns out to be just half the maximum, i.e.

$$
\begin{aligned}
& \eta_{x o}=\frac{1}{2}\left[1-\left(2 \cos ^{2} \phi\right)^{-1}\right], \\
& \eta_{p o}=\frac{1}{2}\left[1-\left(2 \sin ^{2} \phi\right)^{-1}\right] .
\end{aligned}
$$

There are a few special conditions that are of interest. For $\eta=0$ we recover the vacuum state $|x=0\rangle,|n=0\rangle$ whereas for $\eta=1$ we recover the number state $|n=1\rangle$.

\subsection{The mixed Bernoulli state}

We now consider the situation for arbitrary $R$. It is of interest to determine the range of values of $R, \phi$, and $\eta$ for which squeezing occurs, i.e. for which either $(\Delta x)^{2}$ or $(\Delta p)^{2}$ in equations (33) and (34) are less than $\frac{1}{2}$.

It is convenient to transform the parameters $(\eta, \phi, R)$ into three new parameters which are recognized as components of the Bloch vector for this two-level system:

$$
\left.\begin{array}{l}
r_{1}=2 R[\eta(1-\eta)]^{1 / 2} \cos \phi=2^{1 / 2}\langle\hat{x}\rangle \\
r_{2}=2 R[\eta(1-\eta)]^{1 / 2} \sin \phi=2^{1 / 2}\langle\hat{p}\rangle \\
r_{3}=2 \eta-1 .
\end{array}\right\}
$$

We are now in a position to indicate regions of squeezing in the parameter space $\left(r_{1}, r_{2}, r_{3}\right)$. The locus of points of constant $R$ is the ellipsoid of revolution,

$$
\left(r_{1}^{2}+r_{2}^{2}\right) / R^{2}+r_{3}^{2}=1 .
$$


For the pure state $(R=1)$ this becomes a sphere of unity radius. The north and south poles of this sphere correspond to the number state $|n=1\rangle$ and the vacuum state $|0\rangle$ respectively. The phase $\phi$ is the angle that the projection of the vector $\left(r_{1}, r_{2}, r_{3}\right)$ in the $\left(r_{1}, r_{2}\right)$ plane makes with the $r_{1}$ axis.

In figure 3 the regions of squeezing in both $x$ and $p$ are schematically illustrated by the hatched surfaces for two values of $R$. Squeezing can be achieved in the following situations:

(a) when $R=1, r_{3}$ must be negative $\left(\eta<\frac{1}{2}\right)$; the range of permitted values of $\phi$ increases as $r_{3}$ decreases;

(b) for $R<1$, the squeezing range is reduced; $r_{3}$ must be more negative and the range of permitted values of $\phi$ is narrower;

(c) as $R$ is reduced further, a value is reached $\left(R=2^{-1 / 2}\right)$ below which squeezing cannot be attained regardless of the values of the other parameters.

\subsection{Generation of Bernoulli-state light}

The generalized Bernoulli state represents a normalized combination of the zero and one photon states with the appropriate complex coefficients. Random coefficients result in the mixed Bernoulli state. The Bernoulli state should provide a description of the field resulting from 'single-photon emission' from an atom (or a molecule or an ion) when a route for nonradiative decay exists. An example of this kind is discussed below.

Consider a large vessel containing an $\mathrm{N}_{2}$ molecule selectively excited to the $v=1$ level, together with $\mathrm{CO}_{2}$ gas of variable pressure. Vibrationally excited $\mathrm{N}_{2}$ cannot decay by an electric-dipole transition since it is homonuclear; it therefore has a very long lifetime. The molecule can de-excite nonradiatively by collision with a $\mathrm{CO}_{2}$ molecule; indeed this is the preferred method of excitation in $\mathrm{CO}_{2} / \mathrm{N}_{2} / \mathrm{He}$ lasers [7]. The vessel is assumed to be sufficiently large so that wall collisions can be ignored.

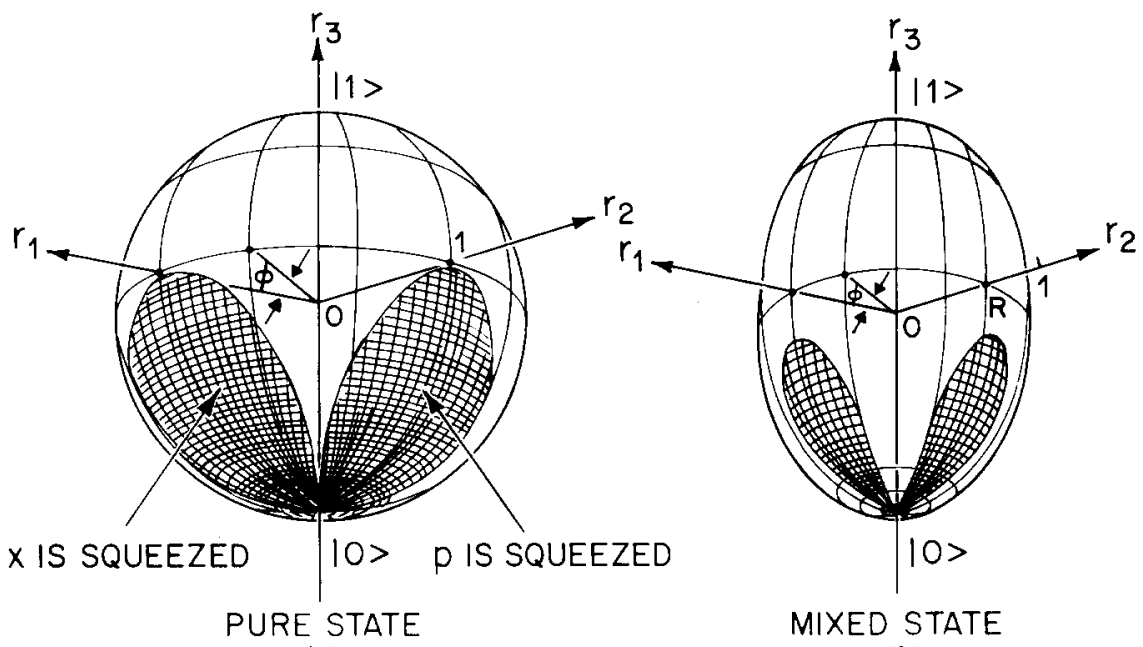

(a)

(b)

Figure 3. Bloch-vector diagram for the Bernoulli state. The left and right lobes of the hatched areas in each figure represent regions of squeezing in $x$ and $p$, respectively. The sphere in $(a)$ represents the pure generalized Bernoulli state. The ellipsoid of revolution in $(b)$ represents a mixed Bernoulli state; the reduced region of squeezing is evident. In both cases, only two of the four lobes representing squeezing are visible from this perspective 
For a $\mathrm{CO}_{2}$ pressure that is vanishingly small the decay is exclusively by photon emission and after a sufficiently long time $\eta \simeq 1$; for a large $\mathrm{CO}_{2}$ pressure the decay is by collision so that $\eta \ll 1$. It is evident that the $\mathrm{CO}_{2}$ pressure may be adjusted to provide any desired value of $\eta$. The radiation at $2359 \mathrm{~cm}^{-1}$ from a single molecule of $\mathrm{N}_{2}$ in this configuration is therefore expected to be in a Bernoulli state. Whether the state is pure or mixed cannot be ascertained without formulating the problem in terms of a hamiltonian.

\section{Discussion}

It has been shown that the binomial state is sub-poissonian, antibunched, and squeezed for certain parameter ranges. Likewise, the mixed Bernoulli state may be squeezed. Therefore, spontaneous photon emission from a single atom may simultaneously exhibit all of these effects under prescribed conditions. Most proposed methods for generating squeezed-state light involve nonlinear optical processes [8]. They are hampered by the complexities and small magnitudes expected from such schemes [9]. A one-photon interaction that leads to a mixed Bernoulli state is provided by the Jaynes-Cummings hamiltonian when the twolevel atom is initially in the excited state and the field is in the vacuum state. That state, however, is not squeezed. Meystre and Zubairy [10] have demonstrated that this system can lead to squeezing when the initial radiation field is in a coherent state. Resonance fluorescence radiation is also expected to simultaneously exhibit these three nonclassical characteristics [11].

Once generated, sub-poissonian light of whatever form is subject to two deleterious effects: Bernoulli random deletion resulting from optical loss (partition noise) and additive independent Poisson noise from the background and the detector. It has been demonstrated that both of these effects dilute the subpoissonian nature of a light beam but fortunately do not destroy it $[12,13]$. Under the effects of random deletion, the binomial photon-counting distribution retains its form but exhibits a variance that increases toward the mean as the deletion becomes stronger. Loudon and Shepherd have similarly shown that absorption and diffraction losses reduce the squeezed nature of a light source; indeed the reduction factor turns out to be the same as that affecting the optical intensity [14]. The antibunching properties of light, on the other hand, are unaffected by random deletion [14].

\section{Acknowledgment}

B. E. A. S. and M. C. T. were supported by the Joint Services Electronics Program (U.S. Army, U.S. Navy, and U.S. Air Force) under contract DAAG29-82K-0080, and by the National Science Foundation under grant ECS-82-19636.

\section{Appendix}

Expansion of coherent state in binomial-state basis

The coherent state $|\alpha\rangle$ may be defined by the condition

$$
\hat{a}|\alpha\rangle=\alpha|\alpha\rangle,
$$

where $\alpha$ is an arbitrary complex number. The expansion of $|\alpha\rangle$ in the number-state basis is well known:

$$
|\alpha\rangle=\exp \left(-|\alpha|^{2} / 2\right) \sum_{n=0}^{\infty} \frac{\alpha^{n}}{\sqrt{ } n !}|n\rangle .
$$


The expansion that we wish to derive is

$$
|\alpha\rangle=\sum_{M=0}^{\infty} C_{M}|\eta, M\rangle
$$

Multiplying equation (A 3 ) on both sides by $\hat{a}$, and using equation (13), gives

$$
\begin{aligned}
\hat{a}|\alpha\rangle & =\sum_{M=1}^{\infty}(\eta M)^{1 / 2} C_{M}|\eta, M-1\rangle \\
& =\sum_{M=0}^{\infty}[\eta(M+1)]^{1 / 2} C_{M+1}|\eta, M\rangle \\
& =\alpha \sum_{M=0}^{\infty} C_{M}|\eta, M\rangle .
\end{aligned}
$$

The last two forms of equation (A 4) yield the recursion relation

$$
[\eta(M+1)]^{1 / 2} C_{M+1}=\alpha C_{M} .
$$

When $\eta \rightarrow 0$ with $M$ finite, all $|\eta, M\rangle$ states become the vacuum state so that the expansion of the coherent state cannot then be accomplished except for the trivial case $|\alpha=0\rangle=|0\rangle$.

The general solution to equation (A 5) is given by

$$
C_{M}=k \frac{(\alpha / \sqrt{ } \eta)^{M}}{\sqrt{ } M !}
$$

where $k(\alpha, \eta, M)$ is an arbitrary constant that is chosen to normalize $|\alpha\rangle$. The desired expansion is therefore

$$
|\alpha\rangle=\sum_{M=0}^{\infty} k(\alpha, \eta, M) \frac{(\alpha / \sqrt{ } \eta)^{M}}{\sqrt{M !}}|\eta, M\rangle .
$$

\section{References}

[1] Giauber, R. J., 1963, Phys. Req., 130, 2529-2539; 1963, Ibid., 131, 27662788.

[2] Milifi, M. M., and Mishkix, E. A., 1967, Phys. Lett. A, 24, 188189.

[3] 'Teich, M. C., Saleh, B. E. A., and Stoler, D., 1983, Optics Commun., 46, 244-248.

[4] Titulaer, U. M., and Glauber, R. J., 1965, Phys. Rev. B, 140, 676-682; 1966,Phys. Rev., 145, 1041-1050.

[5] Stoler, D., 1971, Phys. Rev. D, 4, 2309-2312.

[6] Stoler, D., 1970, Phys. Rev. D, 1, 3217-3219; 1971, Phys. Rer. D, 4, 1925-1926.

[7] Verdeyen, J. T., 1981, Laser Electronics (Prentice-Hall, Englewood-Cliffs, NJ).

[8] WAlls, D. F., 1983, Nature, Lond., 306, 141-146.

[9] Levenson, M. D., 1984, J. Opt. Soc. Am. B, 1, 525.

[10] Meystre, P., and Zubairy, M. S., 1982, Phys. Lett. A, 89, 390-392.

[11] Mandel, L., 1982, Phys. Rev. Lett., 49, 136-138.

[12] Teich, M. C., and Saleh, B. E. A., 1982, Optics Lett., 7, 365-367.

[13] Peřina, J., Saleh, B. E. A., and Teich, M. C., 1983, Optics Commun., 48, 212-214.

[14] Loudon, R., and Shepherd, T. J., 1984, Optica Acta, 31, 1243-1269. 